\title{
Gonado-hypophyseal lesions and reproductive hormonal changes in Brucella melitensis- infected mice and its lipopolysaccharides (LPSs)
}

\begin{abstract}
Gonado-hypophyseal lesions and reproductive hormonal changes in Brucella melitensisinfected mice and its lipopolysaccharide (LPS) were investigated. Forty-eight mice (24 males and 24 females) were used as infection models in this study, and they were divided into three equal separate groups of eight mice based on their sex: M1, M2, and M3 and F1, F2, and F3 (M田Male, F田Flemale). Groups M1 and F1 were orally inoculated with sterile phosphatebuffered saline (PBS) and served as the negative control. Groups M2 and F2 were orally inoculated with $0.4 \mathrm{ml}$ of 109 colony-forming units (cfu) of B. melitensis, while groups M3 and F3 were orally inoculated with $0.4 \mathrm{ml}$ of LPSs from 109 cfu of B. melitensis. All the groups were observed for 10 days for changes in clinical signs or signs of mortality and then euthanized. Whole blood was collected via cardiac puncture, while tissues were fixed in $10 \%$ formalin for histopathological processing. Histopathological lesions in the male and female reproductive tracts ranged from mild to moderate degenerative, inflammatory, and necrotic lesions. These changes were moderate in M2 than in M3 in males and in F3 than in F2 groups in females. Lesions in the pituitary gland ranged from mild to moderate inflammation and necrosis with astrogliosis. F2 and M2 groups had more lesion distribution in the pituitary gland than F3 and M3 groups. There was a $13 \%$ decline in progesterone concentration in F2 and a $44 \%$ decline in F3. Estradiol level declined by $33 \%$ in F2 and slightly increased by 4.2 $\%$ in F3. Progesterone-estrogen ratio (P/E) increased by $31 \%$ in $\mathrm{F} 2$ and declined by $46 \%$ in F3. Testosterone concentration increased by $74 \%$ in M2 and decreased by $56 \%$ in M3. The study showed that oral administration of B. melitensis affects the pituitary gland and may alter the hypothalamic-pituitary-gonadal axis.
\end{abstract}

Keyword: Brucella melitensis; Lipopolysaccharide; Ovaries; Pituitary gland; Reproductive hormones; Testis 